\title{
Virtual Polar Region Method Based on the Earth's Transverse Ellipsoid Model
}

\author{
Feng Wu, ${ }^{1,2}$ Tianyi Shao, ${ }^{1,2}$ Cong Gu, ${ }^{1,2}$ Qiangwen Fu $\mathbb{D}^{\mathbb{D}},{ }^{3}$ and Yafen $\mathrm{Xu}{ }^{1}$ \\ ${ }^{1}$ Shanghai Aerospace Control Technology Institute, Shanghai 201109, China \\ ${ }^{2}$ Shanghai Engineering Research Center of Inertia, Shanghai 201109, China \\ ${ }^{3}$ School of Automation, Northwestern Polytechnical University, Xi'an 710072, China \\ Correspondence should be addressed to Qiangwen Fu; fuqiangwen@nwpu.edu.cn
}

Received 28 November 2020; Revised 22 December 2020; Accepted 19 January 2021; Published 29 January 2021

Academic Editor: Luigi Rodino

Copyright (C) 2021 Feng Wu et al. This is an open access article distributed under the Creative Commons Attribution License, which permits unrestricted use, distribution, and reproduction in any medium, provided the original work is properly cited.

Experimental verification is very important for the research of inertial navigation and integrated navigation technology, but most researchers do not have the opportunity to conduct experiments directly in the polar regions. In order to solve the problem of inertial navigation verification in high latitude areas, a virtual polar region method based on transverse ellipsoid model is proposed. The method converts the reference information, initial state, and inertial sensor data into polar regions based on the transverse geographic coordinate system and can ensure that the attitude, velocity, and altitude information relative to the locallevel frame remain unchanged. Therefore, the actual test data in the middle and low latitudes can be reconstructed accurately in the polar region without singularities, trajectory deformation, and principle errors. Simulation and vehicle tests show that the proposed method can achieve the same verification effect as the actual polar experiment.

\section{Introduction}

Inertial navigation system (INS) has the unique advantages of autonomy, concealment, and information completeness and has become indispensable global navigation equipment for important carriers [1-3]. In the middle and low latitudes, the initial alignment, inertial navigation, and integrated navigation of the INS are usually arranged in the northoriented geographic frame [4-6]. However, there are two kinds of singularities in the polar region of the Earth, which affect the normal work of the INS [7]. One is that the geographic meridians converge rapidly with the increase of latitude, which makes it difficult to solve and express the navigation parameters through the traditional north-oriented mechanization; the other is that the Earth rotation rate and gravity vectors tend to coincide at the two poles of the Earth, which affects the initial alignment process of the INS. Because longitude and latitude are artificially defined, the first kind of influence is called mathematical singularity, which can be solved by designing different mathematical navigation mechanizations; the second kind of influence is physical existence, called physical singularity, which requires higher accuracy inertial sensors or external information assistance to obtain ideal alignment results.

At present, the navigation mechanizations that can work effectively in polar regions include grid mechanization $[8,9]$, transversal Earth mechanization [10-12], pseudo-Earth mechanization $[13,14]$, and wander mechanization $[15,16]$. In addition to navigation mechanization, experimental verification is an important means in the research of inertial navigation and integrated navigation technology. Because the physical field characteristics (including gravity field, Earth's rotation rate, electromagnetic environment, meteorological environment, etc.) in the polar region are obviously different from those in the middle and low latitudes, the economic and political costs of carrying out actual polar navigation experiments are relatively high $[17,18]$. Only some countries and companies have the conditions to conduct polar navigation test directly [19-22].

For most researchers, there is no condition to directly reach the polar region to carry out navigation verification test. The designed polar navigation algorithm can only be 
verified by mathematical simulation method. However, pure mathematical simulation methods are often difficult to truly reflect the motion characteristics of the carrier and the dynamic error of the inertial sensors, which leads to insufficient or even unconvincing verification conclusions. Therefore, it is of great significance to design a virtual polar region technology based on real test data, which can convert the experiment data of middle and low latitude regions into polar regions by mathematical methods for equivalent navigation verification.

Zhou et al. [23] proposed a virtual polar region method using a point on the equator as a new pole, Li and Zang [24] proposed a virtual polar region method using a certain point on the Earth's surface as a new pole, Yao et al. [11] proposed a method to transform the real experiment trajectory to the polar region by using geographic coordinate system, and Lei and $\mathrm{Wu}$ [25] proposed a method of direct coordinate transformation in the Earth-centered Earth-fixed (ECEF) frame to establish the polar trajectory.

In order to overcome the shortcomings of the existing virtual polar method, such as trajectory deformation, model approximation, and limited application area, a virtual polar region method based on transverse ellipsoid model is proposed in this paper. The reference information, initial state, and inertial sensor data are converted to the polar region under the transverse geographic coordinate system so that the motion characteristics of the carrier relative to the local-level frame remain unchanged, which can be applied to any position on the Earth's surface except the transverse poles. In the proposed method, the ellipsoid correction coefficient is used to avoid the spherical approximation and improve the modelling accuracy, so as to achieve the same effect as the actual polar region test.

\section{Transverse Ellipsoid Model}

2.1. Definition of Coordinate System. The relevant coordinate systems involved in this article are defined as follows. The Earth-centered inertial frame is the $i$-frame, in which the $o x_{i}$ and $o y_{i}$ axes are located on the equatorial plane, the $o x_{i}$ axis points to the vernal equinox, the $o z_{i}$ axis points to the north pole of the Earth, and the $o y_{i}$ axis direction is determined according to the right-hand rule. In inertial space, the $o x_{i}$, $o y_{i}$, and $o z_{i}$ coordinate axes point to fixed points and do not rotate with the Earth. The output of inertial sensors is defined in the $i$-frame.

The Earth frame is denoted as the $e$-frame, which is the conventional ECEF frame. The $o x_{e}$ and $o y_{e}$ axes are located in the equatorial plane, the $o x_{e}$ axis points to the Greenwich Meridian, and the $o z_{e}$ axis points to the north pole along the Earth's rotation axis. The three axes constitute the righthand coordinate system. The $e$-frame rotates with the Earth, and the position under the $e$-frame is represented by $\mathbf{p}^{e}=\left[\begin{array}{lll}x_{e} & y_{e} & z_{e}\end{array}\right]^{T}$.

The local-level geographic frame is the $g$-frame, with its three axes pointing east, north, and upward (ENU). The $g$-frame is the computational frame of the traditional northoriented mechanization, and the spherical position under this frame is represented by $\mathbf{p}^{g}=\left[\begin{array}{lll}\lambda & L & h\end{array}\right]^{T}$, where $\lambda, L$, and $h$ denote the longitude, latitude, and altitude, respectively.

The body frame is defined as the $b$-frame, with its three axes pointing right, forward, and upward (RFU).

The transversal Earth frame is the $e_{t}$-frame, which can be obtained by rotating the $e$-frame around $o x_{e}$ axis by $-90^{\circ}$ and then rotating around $o z_{e}$ axis by $-90^{\circ}$. The $o x_{e t}, o y_{e t}$, and $o z_{e t}$ axes of the $e_{t}$-frame coincide with the $o z_{e}, o x_{e}$, and $o y_{e}$ axes of the $e$-frame, respectively, and the pseudo poles, pseudo longitude, and pseudo latitude are constructed, as shown in Figure 1. The position in the $e_{t}$-frame is represented by $\mathbf{p}^{e_{t}}=\left[\begin{array}{lll}x_{t} & y_{t} & z_{t}\end{array}\right]^{T}$.

The transversal geographic frame is the $g_{t}$-frame, which is defined according to the pseudo-longitude and latitude network of the $e_{t}$-frame, with its three axes pointing east, north, and upward of the transverse Earth model. The spherical position under the $g_{t}$-frame is represented by $\mathbf{p}^{g_{t}}=\left[\begin{array}{lll}\lambda_{t} & L_{t} & h_{t}\end{array}\right]^{T}$, where $\lambda_{t}, L_{t}$, and $h_{t}$ denote transverse longitude, latitude, and altitude, respectively.

2.2. Basic Conversion Relationship. According to the definition of the transversal Earth frame in Figure 1, the transformation relationship between the $e$ - and $e_{t}$-frames can be obtained as follows:

$$
\mathbf{C}_{e}^{e_{t}}=\left[\begin{array}{lll}
0 & 0 & 1 \\
1 & 0 & 0 \\
0 & 1 & 0
\end{array}\right]
$$

By derivation, the conversion relationship between the conventional longitude and latitude and the transverse longitude and latitude can be obtained [26]:

$$
\begin{aligned}
& {\left[\begin{array}{l}
\lambda_{t} \\
L_{t}
\end{array}\right]=\left[\begin{array}{c}
a \tan 2(\cos L \cos \lambda, \sin L) \\
a \tan 2\left(\cos L \sin \lambda, \sqrt{1-\cos ^{2} L \sin ^{2} \lambda}\right)
\end{array}\right]} \\
& {\left[\begin{array}{l}
\lambda \\
L
\end{array}\right]=\left[\begin{array}{c}
a \tan 2\left(\sin L_{t}, \cos L_{t} \sin \lambda_{t}\right) \\
a \tan 2\left(\cos L_{t} \cos \lambda_{t}, \sqrt{1-\cos ^{2} L_{t} \cos ^{2} \lambda_{t}}\right)
\end{array}\right],}
\end{aligned}
$$

where $a \tan 2$ is a standard function of the mathematical library, and there is no singular point in its value domain $[-\pi, \pi]$. Considering that both $g$ - and $g_{t}$-frames are locallevel frames, so the upward axes coincide. Obviously, the height information in the two frames is consistent:

$$
h_{t}=h \text {. }
$$

Considering two directional cosine matrices 


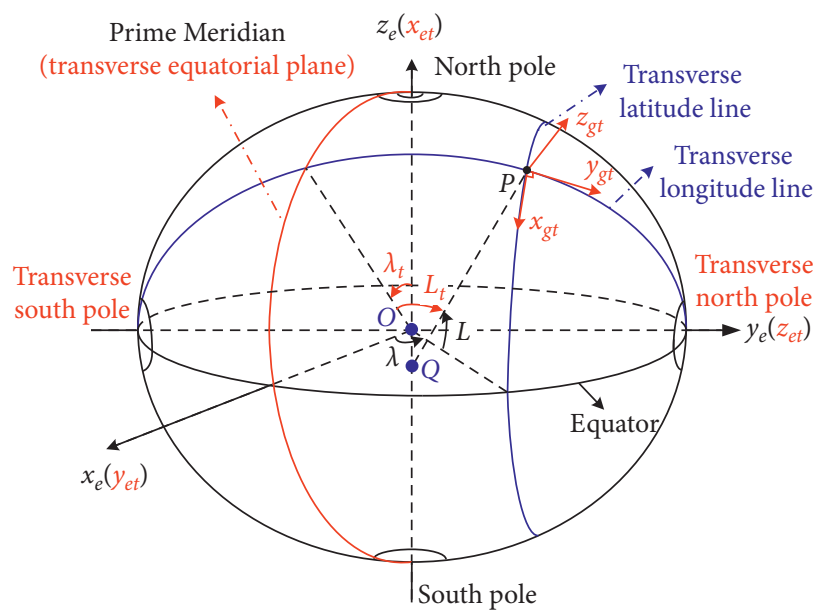

FIgURe 1: Definition of transversal Earth frame.

$$
\begin{aligned}
\mathbf{C}_{g}^{e} & =\left[\begin{array}{ccc}
-\sin \lambda & -\sin L \cos \lambda & \cos L \cos \lambda \\
\cos \lambda & -\sin L \sin \lambda & \cos L \sin \lambda \\
0 & \cos L & \sin L
\end{array}\right], \\
\mathbf{C}_{e_{t}}^{g_{t}} & =\left[\begin{array}{ccc}
-\sin \lambda_{t} & \cos \lambda_{t} & 0 \\
-\sin L_{t} \cos \lambda_{t} & -\sin L_{t} \sin \lambda_{t} & \cos L_{t} \\
\cos L_{t} \cos \lambda_{t} & \cos L_{t} \sin \lambda_{t} & \sin L_{t}
\end{array}\right] .
\end{aligned}
$$

The transformation relation between the $g$ - and $g_{t}$-frames can be obtained as follows:

$$
\mathbf{C}_{g}^{g_{t}}=\mathbf{C}_{e_{t}}^{g_{t}} \mathbf{C}_{e}^{e_{t}} \mathbf{C}_{g}^{e}
$$

Because the $g$ - and $g_{t}$-frames are local-level frames, there is only one transverse azimuth difference $\alpha$ between the two frames.

$$
\mathbf{C}_{g}^{g_{t}}=\left[\begin{array}{ccc}
\cos \alpha & -\sin \alpha & 0 \\
\sin \alpha & \cos \alpha & 0 \\
0 & 0 & 1
\end{array}\right] .
$$

Combining equations (3), (7), and (8), we can get

$$
\left\{\begin{array}{l}
\sin \alpha=\frac{\cos \lambda}{\sqrt{1-\cos ^{2} L \sin ^{2} \lambda}}=\frac{\sin \lambda_{t}}{\sqrt{1-\cos ^{2} L_{t} \cos ^{2} \lambda_{t}}} \\
\cos \alpha=\frac{-\sin L \sin \lambda}{\sqrt{1-\cos ^{2} L \sin ^{2} \lambda}}=\frac{-\sin L_{t} \cos \lambda_{t}}{\sqrt{1-\cos ^{2} L_{t} \cos ^{2} \lambda_{t}}}
\end{array}\right.
$$

where the calculation of $\sin \alpha$ and $\cos \alpha$ seems to have singularity when $\left[\lambda_{t}=0^{\circ}, 180^{\circ} ; L_{t}=0^{\circ}\right]$. However, it can be known from the calculation of conventional longitude $\lambda$ and latitude $L$ that it does not affect the normal calculation.
2.3. Transversal Earth Mechanization. According to the definition of transversal geographic frame, it is easy to get the attitude differential equation in the $g_{t}$-frame as follows:

$$
\dot{\mathbf{C}}_{b}^{g_{t}}=\mathbf{C}_{b}^{g_{t}}\left(\boldsymbol{\omega}_{g_{t} b}^{b} \times\right)=\mathbf{C}_{b}^{g_{t}}\left[\left(\boldsymbol{\omega}_{i b}^{b}-\boldsymbol{\omega}_{i g_{t}}^{b}\right) \times\right] .
$$

The velocity differential equation is

$$
\dot{\mathbf{v}}^{g_{t}}=\mathbf{C}_{b}^{g_{t}} \mathbf{f}^{b}-\left(2 \boldsymbol{\omega}_{i e_{t}}^{g_{t}}+\boldsymbol{\omega}_{e_{t} g_{t}}^{g_{t}}\right) \times \mathbf{v}^{g_{t}}+\mathbf{g}^{g_{t}} .
$$

The differential equations of position direction cosine matrix and height are

$$
\begin{aligned}
\dot{\mathbf{C}}_{e_{t}}^{g_{t}} & =-\left(\boldsymbol{\omega}_{e_{t} g_{t}}^{g_{t}} \times\right) \mathbf{C}_{e_{t}}^{g_{t}}, \\
\dot{h}_{t} & =v_{U}^{g_{t}} .
\end{aligned}
$$

In equations (10)-(13), $\mathbf{C}_{b}^{g_{t}}$ is the transversal attitude matrix, $\mathbf{v}^{g_{t}}$ is the transversal velocity, $\mathbf{C}_{\boldsymbol{e}_{t}}^{g_{t}}$ is the position direction cosine matrix containing the transverse longitude and latitude, and $\boldsymbol{\omega}_{i b}^{b}$ and $\mathbf{f}^{b}$ are the angular rate and specific force output by inertial sensors.

In the differential equations (10)-(13), there are

$$
\begin{aligned}
& \boldsymbol{\omega}_{i g_{t}}^{b}=\mathbf{C}_{g_{t}}^{b}\left(\boldsymbol{\omega}_{i e_{t}}^{g_{t}}+\boldsymbol{\omega}_{e_{t} g_{t}}^{g_{t}}\right), \\
& \boldsymbol{\omega}_{i e_{t}}^{g_{t}}=\mathbf{C}_{e_{t}}^{g_{t}} \boldsymbol{\omega}_{i e_{t}}^{e_{t}}=\mathbf{C}_{e_{t}}^{g_{t}}\left[\begin{array}{lll}
\boldsymbol{\omega}_{i e} & 0 & 0
\end{array}\right]^{T}, \\
& \mathbf{g}^{g_{t}}=\left[\begin{array}{lll}
0 & 0 & -g
\end{array}\right]^{T},
\end{aligned}
$$

where $\omega_{i e}$ is the Earth rotation rate and $g$ is the gravity.

The key of transversal Earth mechanization is to solve the velocity related angular rate. Assuming that the Earth is a sphere of radius $R_{N}$, the angular rate $\boldsymbol{\omega}_{e_{t}}^{g_{t}}$ can be expressed as

$$
\boldsymbol{\omega}_{e_{t} g_{t}}^{g_{t}}=\left[-\frac{v_{N}^{g_{t}^{\prime}}}{R_{N}+h_{t}} \frac{v_{E}^{g_{t}^{\prime}}}{R_{N}+h_{t}} \frac{v_{E}^{g_{t}^{\prime}} \tan L_{t}}{R_{N}+h_{t}}\right]^{T} .
$$


Because the real Earth is an ellipsoid model, the velocity $\mathbf{v}^{g_{t \prime}}$ in equation (17) is not equal to $\mathbf{v}^{g_{t}}$, and there is an ellipsoidal correction coefficient matrix [27]:

$$
\left[\begin{array}{c}
v_{E}^{g_{t}^{\prime}} \\
v_{N}^{g_{t}^{\prime}}
\end{array}\right]=\mathbf{C}_{k}\left[\begin{array}{c}
v_{E}^{g_{t}} \\
v_{N}^{g_{t}}
\end{array}\right]
$$

Since there is no relative motion between the $e$ - and $e_{t}$-frames, that is, $\boldsymbol{\omega}_{e_{t} e}^{g}=\mathbf{0}$, we can obtain the conversion relationship

$$
\begin{aligned}
\boldsymbol{\omega}_{e_{t} g_{t}}^{g_{t}} & =\mathbf{C}_{g}^{g_{t}}\left(\boldsymbol{\omega}_{e_{t} e}^{g}+\boldsymbol{\omega}_{e g}^{g}\right)=\mathbf{C}_{g}^{g_{t}} \boldsymbol{\omega}_{e g}^{g} \\
& =\mathbf{C}_{g}^{g_{t}}\left[-\frac{v_{N}^{g}}{R_{M}+h} \frac{v_{E}^{g}}{R_{N}+h} \frac{v_{E}^{g} \tan L}{R_{N}+h}\right]^{T} .
\end{aligned}
$$

There is an overflow in the calculation of term $\tan L$ in the third element $\omega_{\text {eg, } z}^{g}$ of equation (19). However, according to the composition of matrix $\mathbf{C}_{g}^{g_{t}}$ in formula (8), the element $\omega_{e g, z}^{g}$ does not affect the calculation of horizontal angular rate. After further derivation and simplification, the ellipsoid correction coefficient matrix in equation (18) can be obtained as follows:

$$
\mathbf{C}_{k}=\left[\begin{array}{cc}
1+(k-1) \sin ^{2} \alpha & (1-k) \sin \alpha \cos \alpha \\
(1-k) \sin \alpha \cos \alpha & 1+(k-1) \cos ^{2} \alpha
\end{array}\right],
$$

where $k=\left(R_{N}+h_{t}\right) /\left(R_{M}+h_{t}\right)$ and $R_{M}$ and $R_{N}$ are still the meridian and prime radii of curvature in the conventional $e$-frame.

By adding an ellipsoid correction coefficient $\mathbf{C}_{k}$, the complex process of solving the radii of the transverse meridian and prime circle is avoided in equation (18). Therefore, this method simplifies the algorithm, ensures the accuracy of the model, and avoids principle errors.

\section{Virtual Polar Region Technology}

The influence of polar region on inertial navigation is mainly manifested in two aspects. One is that the Earth rotation rate tends to coincide with the gravity vector; the other is the rapid convergence of meridian. If a point on the Earth has these two characteristics at the same time, it can be called a pole, and the adjacent area is called a polar region. Accordingly, in the inertial sensor data, the Earth rotation rate and gravity value in the polar region can be used to replace the actual measurement value, so as to construct the virtual sensor output.

The ideal virtual polar technology should keep the attitude and velocity information of the vehicle unchanged relative to the local-level frame. In this paper, the polar trajectory reconstruction technology based on the transverse ellipsoid model is designed. The core of the proposed virtual polar region technology is to ensure that the trajectory attitude, velocity, and height information are unchanged relative to the transversal geographic frame during the transformation process. The virtual polar region technology includes three parts: reference information conversion, initial state conversion, and the conversion of inertial measurement unit (IMU) data.

3.1. Reference Information Conversion. The reference information conversion process converts the reference attitude, velocity, and position information to the polar region. In the nonpolar region test, the results of INS/global navigation satellite system (GNSS) integrated navigation in the $g$-frame can be used as reference information, including attitude matrix $\mathbf{C}_{b}^{g}$, velocity $\mathbf{v}^{g}$, and position $\mathbf{p}^{g}$. Firstly, the attitude matrix and velocity reference information are transformed into the $g_{t}$-frame:

$$
\begin{aligned}
& \mathbf{C}_{b}^{g_{t}}=\mathbf{C}_{g}^{g_{t}} \mathbf{C}_{b}^{g}, \\
& \mathbf{v}^{g_{t}}=\mathbf{C}_{g}^{g_{t}} \mathbf{v}^{g},
\end{aligned}
$$

where $\mathbf{C}_{g}^{g_{t}}$ can be solved by equation (7) or (8) according to the position $\mathbf{p}^{g}$.

Since the attitude, velocity, and altitude of the transversal geographic frame need to be kept unchanged in the conversion process, $\mathbf{C}_{b}^{g_{t}}, \mathbf{v}^{g_{t}}$, and height $h$ are directly used as the attitude matrix, velocity, and altitude information of the virtual polar region. Considering the influence of the Earth's curvature, the position information cannot be directly converted, but can be solved by integrating the velocity as follows:

$$
\dot{\mathbf{C}}_{e_{t}}^{g_{t}^{\#}}=-\left(\boldsymbol{\omega}_{e_{t} g_{t}}^{g_{t}^{\#}} \times\right) \mathbf{C}_{e_{t}}^{g_{t}^{\#}}
$$

where the superscript "\#” denotes the relevant parameters of the reconstructed trajectory, so as to distinguish it from the actual experiment data; $\mathbf{C}_{e_{t}}^{g_{t}^{\#}}$ is the position matrix containing transverse longitude and latitude, and its initial value $\mathbf{C}_{e_{t}(0)}^{g_{t}^{\#}}$ can be obtained from the initial state conversion; $\boldsymbol{\omega}_{e_{t} g_{t}}^{g_{t}^{\#}}$ is the angular rate related to position change, which is calculated by equation (17) according to the velocity $\mathbf{v}^{g_{t}}$ and the reconstructed transverse longitude and latitude.

According to the position matrix $\mathbf{C}_{e_{t}}^{g_{t}^{\#}}$, the transverse longitude and latitude of virtual polar region can be obtained:

$$
\left[\begin{array}{c}
\lambda_{t}^{\#} \\
L_{t}^{\#}
\end{array}\right]=\left[\begin{array}{c}
a \tan 2\left(-C_{11}, C_{12}\right) \\
a \tan 2\left(C_{33}, C_{23}\right)
\end{array}\right]
$$

where $C_{i j}$ represents the element in the $i$-th row and $j$-th column of matrix $\mathbf{C}_{e_{t}}^{g_{t}^{\#}}$. After obtaining the transverse longitude $\lambda_{t}^{\#}$ and latitude $L_{t}^{\#}$, the traditional longitude $\lambda^{\#}$ and latitude $L^{\#}$ of the virtual polar region can be calculated according to equation (3).

3.2. Initial State Conversion. The initial position setting is the key to the virtual polar region technology. The starting position is artificially set to a certain point in the polar region, so the subsequent virtual polar region trajectory can be obtained by integrating from this starting point. The 
initial position point $\mathbf{p}_{0}^{g}$ of the polar region is converted to the $g_{t}$-frame to get $\mathbf{p}_{0}^{g_{t}}$ :

$$
\mathbf{p}_{0}^{g_{t}}=\left[\begin{array}{l}
\lambda_{0 t} \\
L_{0 t} \\
h_{0 t}
\end{array}\right]=\left[\begin{array}{c}
a \tan 2\left(\cos L_{0} \cos \lambda_{0}, \sin L_{0}\right) \\
a \tan 2\left(\cos L_{0} \sin \lambda_{0}, \sqrt{1-\cos ^{2} L_{0} \sin ^{2} \lambda_{0}}\right) \\
h_{0}
\end{array}\right],
$$

where $\lambda_{0}, L_{0}$, and $h_{0}$ are the artificially designated initial longitude, latitude, and altitude, and then the initial position matrix $\mathbf{C}_{e_{t}(0)}^{g_{t}^{\#}}$ can be obtained by equation (6).

The conversion methods of the initial attitude matrix and the initial velocity are the same as equations (21) and (22).

3.3. IMU Data Conversion. IMU data conversion process is to convert the angular rate measured by gyros and the specific force measured by accelerometers to the polar region. The reconstruction of gyroscope and accelerometer data in the polar region is to subtract the local Earth rotation rate and gravity vectors in the transversal geographic frame and then add the corresponding vectors of the virtual polar region. The conversion of gyro angular rate is

$$
\widetilde{\boldsymbol{\omega}}_{i b}^{b \#}=\widetilde{\boldsymbol{\omega}}_{i b}^{b}-\mathbf{C}_{g_{t}}^{b} \boldsymbol{\omega}_{i g_{t}}^{g_{t}}+\mathbf{C}_{g_{t}}^{b \#} \boldsymbol{\omega}_{i g_{t}}^{g_{t}^{\#}}=\widetilde{\boldsymbol{\omega}}_{i b}^{b}+\mathbf{C}_{g_{t}}^{b}\left(\boldsymbol{\omega}_{i g_{t}}^{g_{t}^{\#}}-\boldsymbol{\omega}_{i g_{t}}^{g_{t}}\right)
$$

where $\boldsymbol{\omega}_{i g_{t}}^{g_{t}}$ is the local angular rate, $\boldsymbol{\omega}_{i g_{t}}^{g_{t}^{\#}}$ is the converted polar angular rate, which can be solved by referring to equation (14), and $\widetilde{\boldsymbol{\omega}}_{i b}^{b}$ is the actual output of the gyro angular rate.

The conversion method of the specific force measured by the accelerometers is

$$
\begin{aligned}
\widetilde{\mathbf{f}}^{b \#} & =\widetilde{\mathbf{f}}^{b}-\mathbf{C}_{g_{t}}^{b}\left[\left(2 \boldsymbol{\omega}_{i e}^{g_{t}}+\boldsymbol{\omega}_{e g_{t}}^{g_{t}}\right) \times \mathbf{v}^{g_{t}}-\mathbf{g}^{g_{t}}\right]+\mathbf{C}_{g_{t}}^{b \#}\left[\left(2 \boldsymbol{\omega}_{i e}^{g_{t}^{\#}}+\boldsymbol{\omega}_{e g_{t}}^{g_{t}^{\#}}\right) \times \mathbf{v}^{g_{t}^{\#}}-\mathbf{g}^{g_{t}^{\#}}\right] \\
& =\widetilde{\mathbf{f}}^{b}+\mathbf{C}_{g_{t}}^{b}\left[\left(2 \boldsymbol{\omega}_{i e}^{g_{t}^{\#}}+\boldsymbol{\omega}_{e g_{t}}^{g_{t}^{\#}}-2 \boldsymbol{\omega}_{i e}^{g_{t}}-\boldsymbol{\omega}_{e g_{t}}^{g_{t}}\right) \times \mathbf{v}^{g_{t}}-\left(\mathbf{g}^{g_{t}^{\#}}-\mathbf{g}^{g_{t}}\right)\right]
\end{aligned}
$$

where $\widetilde{\mathbf{f}}^{b}$ is the actual output of the accelerometer; $\boldsymbol{\omega}_{i e}^{g_{t}}, \boldsymbol{\omega}_{e g_{t}}^{g_{t}}$, and $\mathbf{g}^{g_{t}}$ are calculated according to the local velocity and position information; $\boldsymbol{\omega}_{i e}^{g_{t}^{\#}}, \boldsymbol{\omega}_{e g_{t}}^{g_{t}^{\#}}$, and $\mathbf{g}_{t}^{g_{t}^{\#}}$ are solved according to the velocity and position information converted to the polar region.

\section{Verification Results}

4.1. Simulation Test. Firstly, the proposed virtual polar technology is verified by simulation. In the simulated trajectory, the starting point is set as $\left[120^{\circ} \mathrm{E}, 50^{\circ} \mathrm{N}, 0 \mathrm{~m}\right]$, and the ship travels north along the $120^{\circ} \mathrm{E}$ meridian at a speed of $10 \mathrm{~m} / \mathrm{s}$ for 48 hours without attitude and altitude change. The simulated nonpolar data is used to reconstruct the polar trajectory, and the starting point of the polar trajectory is placed at the North Pole. Figure 2 shows the original trajectory and the reconstructed polar trajectory curves. From the trajectory shape, the proposed virtual polar technology can accurately reproduce the nonpolar test trajectory in the polar region.

In addition to the shape of the virtual polar trajectory, the key is to verify whether the virtual polar trajectory satisfies the dynamic differential equations of the INS. Without adding any error, the transversal Earth mechanization is used to verify the navigation solution of the virtual polar trajectory, and the results are shown in Figure 3.

It can be seen from Figure 3 that the attitude error, velocity error, and position error of the pure inertial navigation for the reconstructed polar trajectory are within $5 e-8^{\circ}, 5 e-6 \mathrm{~m} / \mathrm{s}$, and $0.05 \mathrm{~m}$, respectively, which are very small and mainly caused by computer rounding errors. These results show that the reconstructed polar trajectory satisfies the dynamics model and has no principle error, so it can be used as the verification trajectory of the INS.

4.2. Vehicle Test. The effectiveness of virtual polar technology is further verified by a vehicle navigation test. The onboard test platform consists of a domestic optical fiber IMU, a barometric altimeter, and an OEM615 single antenna GNSS receiver. Among them, the gyroscope accuracy is $0.02 \%$, the accelerometer accuracy is $40 \mu \mathrm{g}$, and the data output frequency is $200 \mathrm{~Hz}$. The barometric altimeter provides $30 \mathrm{~m}$ altitude information to damp the vertical channel of pure inertial navigation. The GNSS receiver has a velocity measurement accuracy of $0.1 \mathrm{~m} / \mathrm{s}$ and a positioning accuracy of $5 \mathrm{~m}$.

The vehicle test uses a driving route from Nanjing, China to Suzhou, with a working time of $6.1 \mathrm{~h}$ and a driving range of about $300 \mathrm{~km}$. In Figure 4, the blue curve represents the actual test route with the starting point at $\left[118.76^{\circ} \mathrm{E}\right.$, $31.89^{\circ} \mathrm{N}$ ], and the red curve represents the reconstructed polar trajectory. The starting point of the polar trajectory is artificially set at $\left[148.76^{\circ} \mathrm{E}, 89^{\circ} \mathrm{N}\right]$ so that the middle section of the trajectory passes through the vicinity of the North Pole. Since the virtual polar region method guarantees that the velocity and attitude information relative to the local horizontal plane remain unchanged, from the trajectory shape, the virtual polar region trajectory reproduces the real test trajectory very well.

The transversal Earth mechanization is used to complete the pure inertial navigation for the actual test data and the virtual polar trajectory, and the transverse longitude and latitude errors are shown in Figure 5. It can be seen from the figure that the navigation position error of the virtual polar 


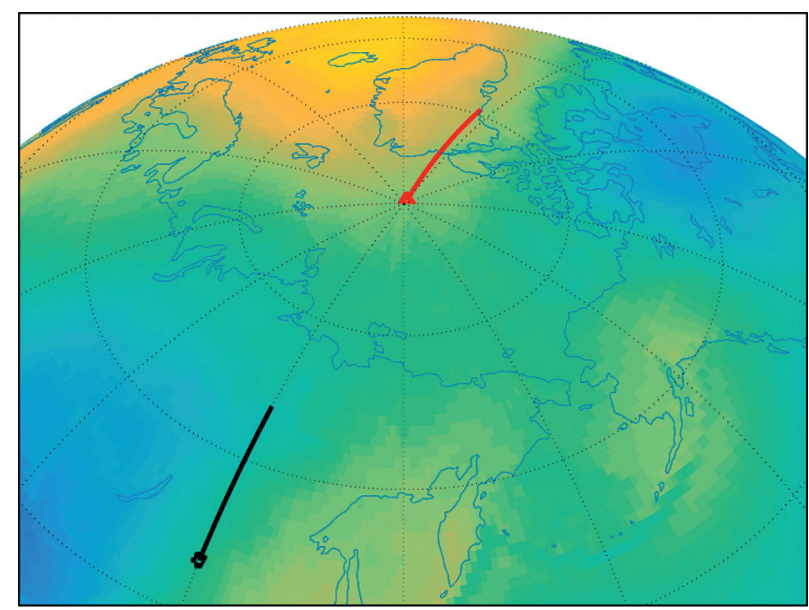

- Original starting point

$\Delta \quad$ Virtualized starting point

- Original nonpolar route

_ Virtualized polar route

FIGURE 2: Simulated nonpolar route and virtualized polar trajectory.

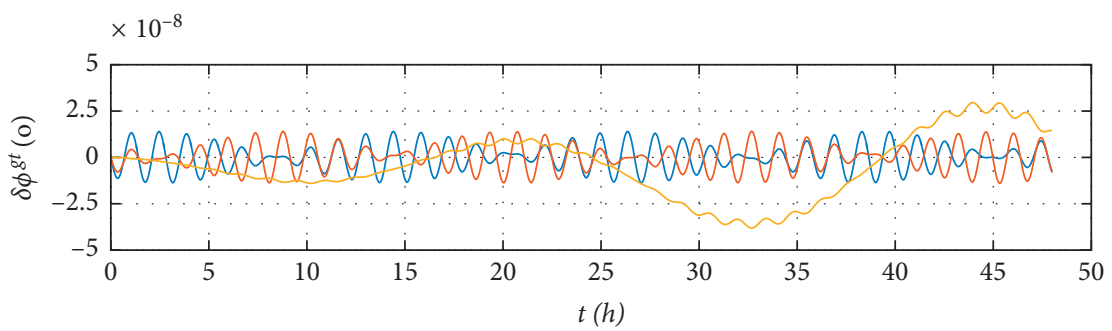

$-\delta \phi_{E}^{g t}$

$-\delta \phi_{N}^{g t}$

$-\delta \phi_{U}^{g t}$

(a)

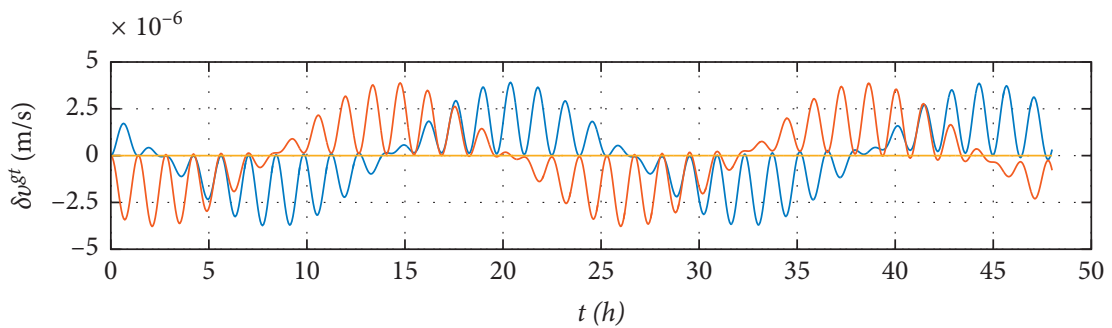

$-\delta v_{E}^{g t}$

$-\delta v_{N}^{g t}$

$-\delta v_{U}^{g t}$ 


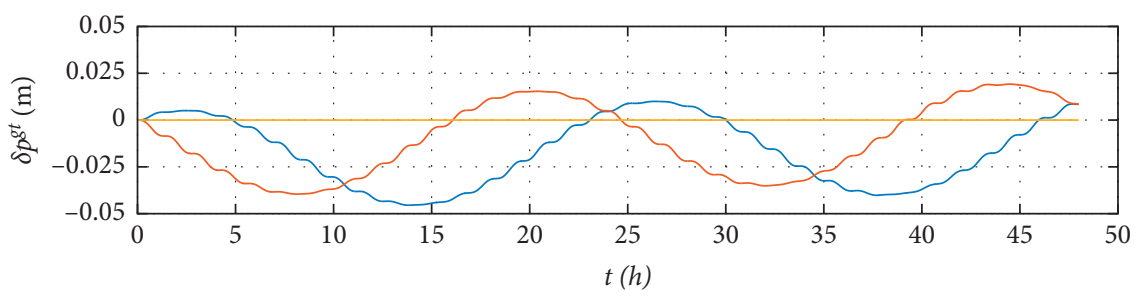

$-\delta \lambda_{t}$
$-\delta L_{t}$
$-\delta h_{t}$

(c)

FiguRE 3: Pure inertial navigation results for the virtualized polar trajectory.
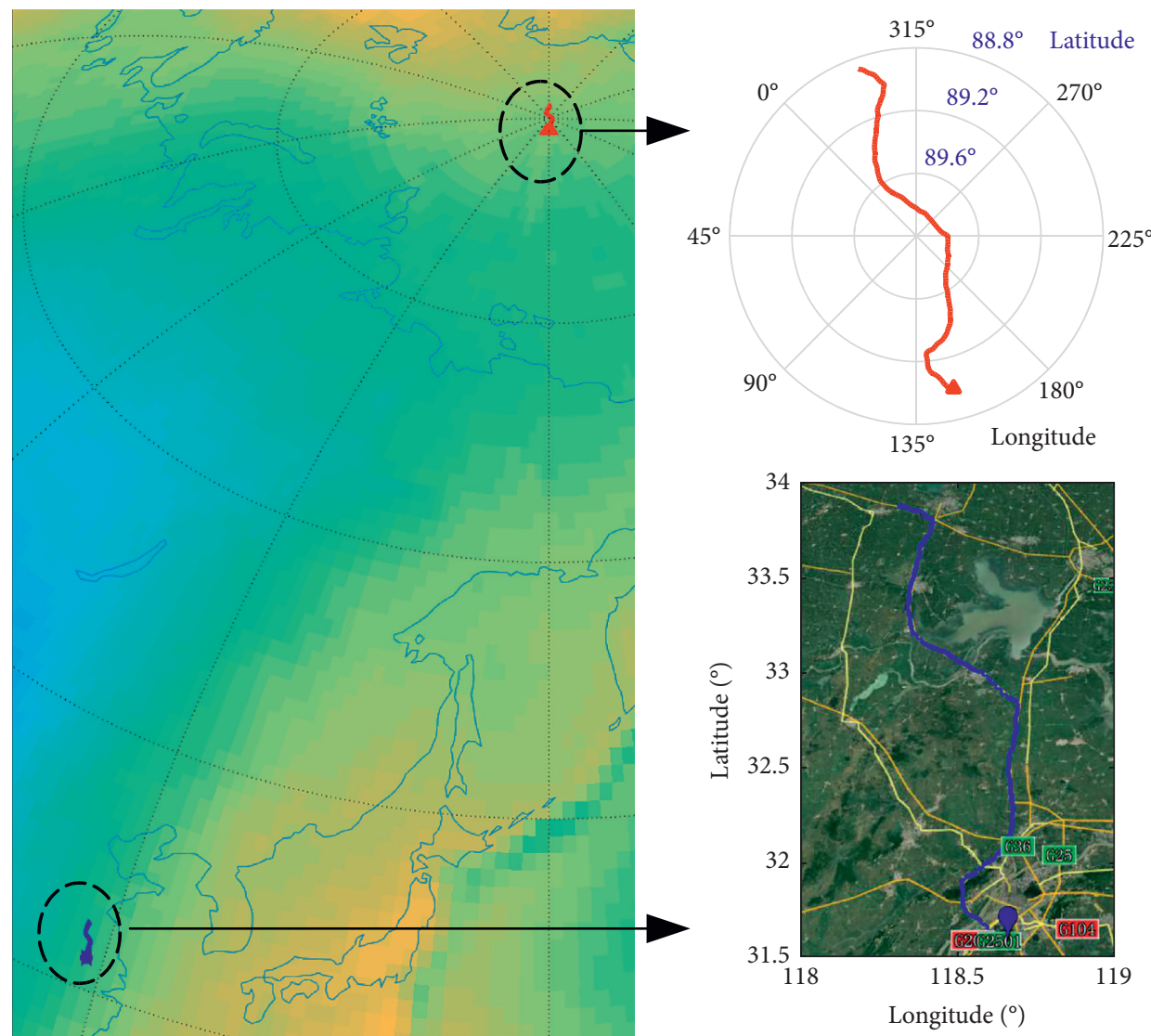

Actual starting point

- Actual route

A Polar staring point

Virtualized polar route

FIGURE 4: Vehicle test path and virtualized polar trajectory. 


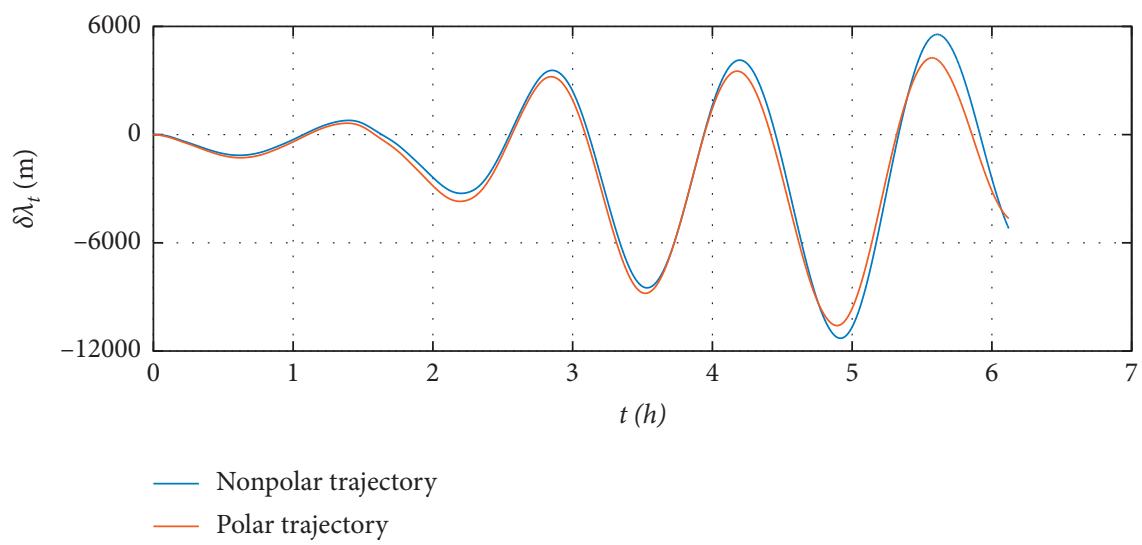

(a)

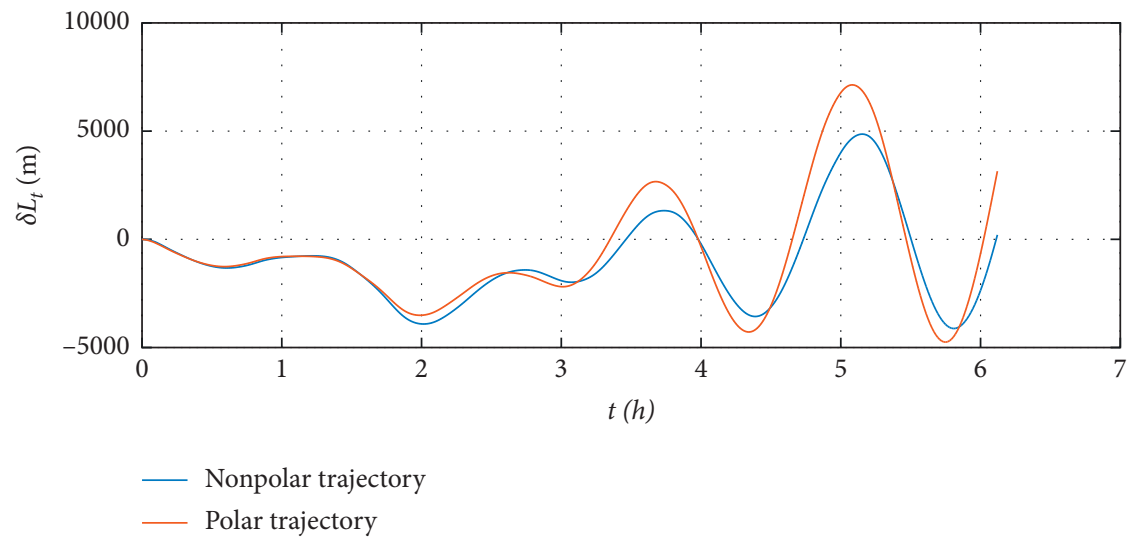

(b)

Figure 5: Navigation position errors of the vehicle test and the virtual polar trajectory.

trajectory is smooth without sudden change, and the magnitude of the error is equivalent to that of actual trajectory. According to the transversal error propagation equations, although the initial error and sensor error between the real trajectory and the virtual polar trajectory are the same, the navigation results are also affected by latitude, gravity, and angular rate, so the navigation errors of the two trajectories are not completely coincident. This is consistent with the real situation. The navigation results of the INS with the same error must be different in different positions.

\section{Conclusions}

This paper proposes a virtual polar region technology based on the transverse ellipsoid model, which can ensure that the attitude, velocity, and height information of the vehicle relative to the transversal geographic frame remain unchanged, and the polar trajectory can be reconstructed accurately according to the actual test data in the middle and low latitudes. The method can solve the problem of difficulty in polar region navigation verification and can achieve the same effect as the actual polar test. The virtualized polar trajectory is only used to verify the pure inertial navigation in this paper, and it can be further used to verify the initial alignment and integrated navigation algorithms.

\section{Data Availability}

The raw/processed data required to reach the findings in this paper cannot be shared at this time as the data also form part of an ongoing study.

\section{Conflicts of Interest}

The authors declare that there are no conflicts of interest regarding the publication of this paper.

\section{References}

[1] G. Hu, W. Wang, Y. Zhong, B. Gao, and C. Gu, "A new direct filtering approach to INS/GNSS integration," Aerospace Science and Technology, vol. 77, pp. 755-764, 2018.

[2] Q. Fu, S. Li, Y. Liu, and F. Wu, "Information-reusing alignment technology for rotating inertial navigation system," Aerospace Science and Technology, vol. 99, Article ID 105747, 8 pages, 2020.

[3] Q. Zhou, All-Earth Inertial Navigation Algorithm for Large Aircraft, Northwestern Polytechnical University, Xi'an, China, 2013.

[4] B. Zhu, J. Xu, H. He, W. An, and Y. Liang, "Analysis of the influence of initial positioning error on SINS in-motion alignment," Flight Control and Detection, vol. 2, no. 6, pp. $48-53,2019$. 
[5] J. Zhao, C. Cui, and J. Zhu, "A robust double-stage EKF design for navigation system of micro UAVs based on 9-D MIMU/ GPS," Flight Control and Detection, vol. 2, no. 2, pp. 1-9, 2019.

[6] G. Hu, B. Gao, Y. Zhong, and C. Gu, "Unscented Kalman filter with process noise covariance estimation for vehicular INS/ GPS integration system," Information Fusion, vol. 64, pp. 194-204, 2020.

[7] Q. Fu, Q. Zhou, G. Yan, S. Li, and F. Wu, "Unified all-Earth navigation mechanization and virtual polar region technology," IEEE Transactions on Instrumentation and Measurement, vol. 70, pp. 1-11, 2021.

[8] M. B. Ignagni, "An all-Earth inertial navigation scheme," Navigation, vol. 19, no. 3, pp. 209-214, 1972.

[9] Q. Zhou, Y. Qin, Q. Fu, and Y. Yue, "Grid mechanization in inertial navigation systems for transpolar aircraft," Journal of Northwestern Polytechnical University, vol. 31, no. 2, pp. 210-217, 2013.

[10] W. K. Lyon, "The navigation of arctic polar submarines," Journal of Navigation, vol. 37, no. 2, pp. 155-179, 1984.

[11] Y.-q. Yao, X.-s. Xu, Y. Li, Y.-t. Liu, J. Sun, and J.-w. Tong, "Transverse navigation under the ellipsoidal earth model and its performance in both polar and non-polar areas," Journal of Navigation, vol. 69, no. 2, pp. 335-352, 2015.

[12] Q. Li, Y. Ben, F. Yu, and J. Tan, "Transversal strapdown INS based on reference ellipsoid for vehicle in the polar region," IEEE Transactions on Vehicular Technology, vol. 65, no. 9, pp. 7791-7795, 2016.

[13] M. Liu, Y. Gao, G. Li, X. Guang, and S. Li, “An improved alignment method for the strapdown inertial navigation system (SINS)," Sensors, vol. 16, p. 17, Article ID 621, 2016.

[14] M. Liu, G. Li, Y. Gao, S. Li, Q. Meng, and S. Du, "Improved polar inertial navigation algorithm based on pseudo INS mechanization," Aerospace Science and Technology, vol. 77, pp. 105-116, 2018.

[15] G. D. Core and V. Nastro, "A world-wide mechanization in inertial navigation systems," Journal of Navigation, vol. 39, no. 3, pp. 441-445, 1986.

[16] Q. Zhou, Y. Yue, X. Zhang, and Y. Tian, "Indirect grid inertial navigation mechanization for transpolar aircraft," Journal of Chinese Inertial Technology, vol. 22, no. 1, pp. 18-22, 2014.

[17] Y. Zhao, Y. Ben, and Q. Li, "Inertial navigation systems for polar marine navigation: a survey," Navigation Positioning and Timing, vol. 7, no. 2, pp. 1-10, 2020.

[18] L. Song, G. Yang, W. Zhao, Y. Ding, and F. Wu, "The inertial integrated navigation algorithms in the polar region," Mathematical Problems in Engineering, vol. 2020, Article ID 5895847, 9 pages, 2020.

[19] N. Fischer, B. B. Hardy, C. Johnson, and J. Wail, "Polar flight test of CGEM GPS in an integrated navigation system on a KC-10," in Proceedings of International Technical Meeting of the Satellite Division of the Institute of Navigation, Kansas City, MO, USA, September 1997.

[20] A. G. Andreev, V. S. Ermakov, M. B. Mafter, V. I. Kokorin, and C. V. Rumyantsev, "High latitude trials of modern Russian marine compasses," in Proceedings of 2006 IEEE/ION Position, Location, and Navigation Symposium, Coronado, CA, USA, April 2006.

[21] A. G. Andreev, V. S. Ermakov, M. B. Mafter, V. I. Kokorin, and C. V. Rumiancev, "Results of high latitude trials of modern marine compasses," IEEE Aerospace and Electronic Systems Magazine, vol. 20, no. 10, pp. 29-31, 2005.

[22] Y. Paturel, J. B. Lacambre, F. Patin, and C. Moynagh, "Inertial navigation at high latitude: trials and test results," in
Proceedings of OCEANS, MTS/IEEE, Washington, DC, USA, October 2015.

[23] Q. Zhou, M. Zhao, and Y. Tian, "Verification of polar navigation elements based on fictions pole of earth," in Proceedings of IEEE Chinese Guidance, Navigation and Control Conference, IEEE, Nanjing, China, August 2016.

[24] L. Li and H. Zhang, "Study on application of virtual polar technique in inertial navigation systems polar sailing," in Proceedings of Development and Application of Optical Gyroscope and System Technology, Jiujiang, China, September 2016.

[25] J. Lei and W. Wu, "Simulation and algorithm verification for Polar Region inertial navigation based on low latitude test sailing," in Proceedings of AsiaSim/SCS AutumnSim 2016, Beijing, China, October 2016.

[26] X. Xu and M. Dou, "Inertial navigation algorithm in polar regions based on transverse geographic coordinate system," Journal of Huazhong University of Science and Technology, vol. 42, no. 12, pp. 116-121, 2014.

[27] F. Qin, L. Chang, L. Tong, Z. Wang, C. Huang, and F. Zha, "Transverse polar navigation method based on virtual sphere model," Journal of Chinese Inertial Technology, vol. 26, no. 5, pp. 571-578, 2018. 